\title{
Article \\ Coefficient Bounds for Certain Classes of Analytic Functions Associated with Faber Polynomial
}

\author{
Adel A. Attiya ${ }^{1,2}$, Abdel Moneim Lashin ${ }^{2,3} \mathbb{D}$, Ekram E. Ali ${ }^{1,4} \mathbb{D}^{\mathrm{D}}$ and Praveen Agarwal ${ }^{5,6,7,8, *}$ \\ 1 Department of Mathematics, College of Science, University of Ha'il, Ha'il 81451, Saudi Arabia; \\ aattiy@mans.edu.eg (A.A.A.); ekram_008eg@yahoo.com (E.E.A.) \\ 2 Department of Mathematics, Faculty of Science, Mansoura University, Mansoura 35516, Egypt; \\ aylashin@mans.edu.eg \\ 3 Department of Mathematics, Faculty of Science, King Abdulaziz University, Jeddah 21589, Saudi Arabia \\ 4 Department of Mathematics and Computer Science, Faculty of Science, Port Said University, \\ Port Said 42521, Egypt \\ 5 Department of Mathematics, Anand International College of Engineering, Jaipur 303012, India \\ 6 International Center for Basic and Applied Sciences, Jaipur 302029, India \\ 7 Department of Mathematics, Harish-Chandra Research Institute, Allahabad 211 019, India \\ 8 Department of Mathematics, Netaji Subhas University of Technology, New Delhi 110078, India \\ * Correspondence: praveen.agarwal@anandice.ac.in or goyal.praveen2011@gmail.com
}

check for

updates

Citation: Attiya, A.A.; Lashin, A.M.; Ali, E.E.; Agarwal, P. Coefficient Bounds for Certain Classes of Analytic Functions Associated with Faber Polynomial. Symmetry 2021, 13, 302. https://doi.org/10.3390/ sym 13020302

Academic Editor: Dmitriy V. Dolgy Received: 31 October 2020 Accepted: 30 January 2021 Published: 10 February 2021

Publisher's Note: MDPI stays neutral with regard to jurisdictional clai$\mathrm{ms}$ in published maps and institutional affiliations.

Copyright: $(\odot 2021$ by the authors. Licensee MDPI, Basel, Switzerland. This article is an open access article distributed under the terms and conditions of the Creative Commons Attribution (CC BY) license (https:// creativecommons.org/licenses/by/ $4.0 /)$.

\begin{abstract}
In this paper, we intorduce a family of analytic functions in the open unit disk which is bi-univalent. By the virtue of the Faber polynomial expansions, the estimation of $n-t h(n \geq 3)$ Taylor-Maclaurin coefficients $\left|a_{n}\right|$ is obtained. Furthermore, the bounds value of the first two coefficients of such functions is established.
\end{abstract}

Keywords: faber polynomial; coefficient bounds; uniformly convex; uniformly starlike; univalent functions; bi-univalent functions

\section{Introduction}

Faber polynomials, which were introduced by Faber in 1903 [1], play an important role in the theory of functions of a complex variable and different areas of mathematics and there is a rich literature [2-7] describing their properties and their applications. Given a function $h(z)$ of the form

$$
h(z)=z+b_{0}+b_{1} z^{-1}+b_{2} z^{-2}+\ldots,
$$

consider the expansion

$$
\frac{s h^{\prime}(\zeta)}{h(\zeta)-w}=\sum_{n=0}^{\infty} \Psi_{n}(w) \zeta^{-n}
$$

valid for all $\zeta$ in some neighborhood of $\infty$. The function $\Psi_{n}(w)=w^{n}+\sum_{k=1}^{n} a_{n k} w^{n-k}$ is a polynomial of degree $n$, called the $n$-th Faber polynomial with respect to the function $h(z)$. In particular,

$$
\begin{aligned}
& \Psi_{0}(w)=1, \quad \Psi_{1}(w)=w-b_{0} \\
& \Psi_{2}(w)=w^{2}-2 b_{0} w+\left(b_{0}^{2}-2 b_{1}\right) \\
& \Psi_{3}(w)=w^{3}-3 b_{0} w^{2}+\left(3 b_{0}^{2}-3 b_{1}\right) w+\left(b_{0}^{3}+3 b_{1} b_{0}-3 b_{2}\right) .
\end{aligned}
$$

Let $\Psi_{n}(0)=F_{n}\left(b_{0}, b_{1}, \ldots, b_{n}\right), n \geq 0$, see ([8], p. 118). Let $A$ denote the class of all functions of the form:

$$
f(z)=z+\sum_{n=2}^{\infty} a_{n} z^{n},
$$


which are analytic in the open unit disc $U=\{z: z \in \mathbb{C}$ and $|z|<1\}$ and let $S$ be the class of all functions in $A$ which are univalent in $U$. By using the Faber polynomial expansion of functions of the form (1), Airault and Bouali [9], p. 184 showed that

$$
\frac{z f^{\prime}(z)}{f(z)}=1-\sum_{j=2}^{\infty} F_{j-1}\left(a_{2}, a_{3}, \ldots, a_{j}\right) z^{j-1},
$$

where $F_{j-1}\left(a_{2}, a_{3}, \ldots, a_{j}\right)$ is the Faber polynomial given by:

$$
F_{j-1}\left(a_{2}, a_{3}, \ldots, a_{j}\right)=\sum_{i_{1}+2 i_{2}+\ldots+(j-1) i_{j-1}=j-1} A\left(i_{1}, i_{2}, \ldots, i_{j-1}\right)\left(a_{2}^{i_{1}}, a_{3}^{i_{2}}, \ldots, a_{j}^{i_{j-1}}\right)
$$

and

$$
A\left(i_{1}, i_{2}, \ldots, i_{j-1}\right):=(-1)^{(j-1)+2 i_{1}+\ldots+j i_{j-1}} \frac{\left(i_{1}+i_{2}+\ldots+i_{j-1}-1\right) !(j-1)}{\left(i_{1}\right) !\left(i_{2}\right) ! \ldots\left(i_{j-1}\right) !} .
$$

The first few terms of the Faber polynomials $F_{j-1}, j \geq 2$, are given by (e.g., see [10], p. 52)

$$
\begin{aligned}
& F_{1}=-a_{2} \\
& F_{2}=a_{2}^{2}-2 a_{3} \\
& F_{3}=-a_{2}^{3}+3 a_{2} a_{3}-3 a_{4} \\
& F_{4}=a_{2}^{4}-4 a_{2}^{2} a_{3}+4 a_{2} a_{4}+2 a_{3}^{2}-4 a_{5} \\
& F_{5}=-a_{2}^{5}+5 a_{2}^{3} a_{3}+5 a_{2}^{2} a_{4}-5 a_{2}\left(a_{3}^{2}-a_{5}\right)+5 a_{3} a_{4}-5 a_{6} .
\end{aligned}
$$

The Koebe one-quarter theorem [8], p. 31 ensures the range of every function of the class $S$ contains the disc $\left\{w:|w|<\frac{1}{4}\right\}$. Thus every univalent function $f \in S$ has an inverse $f^{-1}$, which is defined by

$$
f^{-1}(f(z))=z \quad(z \in U)
$$

and

$$
f\left(f^{-1}(\omega)\right)=\omega \quad\left(|\omega|<\frac{1}{4}\right) .
$$

The inverse map $g:=f^{-1}$ of the function $f \in A$ has Taylor expansion given by (see [9], p. 185)

$$
\begin{aligned}
g(\omega) & =f^{-1}(\omega)=w+\sum_{n=2}^{\infty} \frac{1}{n} K_{n-1}^{-n}\left(a_{2}, a_{3}, \ldots, a_{n}\right) \omega^{n} \\
& =w-a_{2} \omega^{2}+\left(2 a_{2}^{2}-a_{3}\right) \omega^{3}-\left(5 a_{2}^{2}-5 a_{2} a_{3}+a_{4}\right) \omega^{4}+\ldots,
\end{aligned}
$$


where the coefficients $K_{n}^{p}\left(a_{2}, a_{3}, \ldots, a_{n}\right)$ are given by

$$
\begin{aligned}
K_{1}^{p} & =p a_{2}, \quad K_{2}^{p}=\frac{p(p-1)}{2} a_{2}^{2}+p a_{3}, \\
K_{3}^{p} & =p(p-1) a_{2} a_{3}+p a_{4}+\frac{p(p-1)(p-2)}{3 !} a_{2}^{3}, \\
K_{4}^{p} & =p(p-1) a_{2} a_{4}+p a_{5}+\frac{p(p-1)}{2} a_{3}^{2}+\frac{p(p-1)(p-2)}{2} a_{2}^{2} a_{3}+\frac{p !}{(p-4) ! 4 !} a_{2}^{4}, \\
& \vdots \\
K_{n}^{p} & =\frac{p !}{(p-n) ! n !} a_{2}^{n}+\frac{p !}{(p-n+1) !(n-2) !} a_{2}^{n-2} a_{3}+\frac{p !}{(p-n+2) !(n-3) !} a_{2}^{n-3} a_{4} \\
& +\frac{p !}{(p-n+3) !(n-4) !} a_{2}^{n-4}\left[a_{5}+\frac{p-n+3}{2} a_{3}^{2}\right] \\
& +\frac{p !}{(p-n+4) !(n-5) !} a_{2}^{n-4}\left[a_{6}+(p-n+3) a_{3} a_{4}\right]+\sum_{j \geq 6}^{\infty} a_{2}^{n-j} V_{j}
\end{aligned}
$$

and $V_{j}$ is homogeneous polynomial of degree $j$ in the variables $a_{3}, \ldots, a_{n}$, see ([11], p. 349 and [9], p. 183 and p. 205).

Lemma 1. (Schwarz lemma [8], $p$. 3) Let $\omega(z)$ be analytic in the unit disc $U$, with $\omega(0)=0$ and $|\omega(z)|<1$ in $U$. Then $|\omega(z)|<|z|$ and $\left|\omega^{\prime}(0)\right|<1$ in $U$.

If $f$ and $g$ are analytic functions in $U$, we say that $f$ is subordinate to $g$, written $f(z) \prec g(z)$ if there exists a Schwarz function $\omega(z)$ such that $f(z)=g(\omega(z))$. Let $\phi$ be an analytic function with positive real part in $U$, satisfying $\phi(0)=1, \phi^{\prime}(0)>0$, and $\phi(U)$ is symmetric with respect to the real axis. Such a function has a Taylor series of the form

$$
\phi(z)=1+B_{1} z+B_{2} z^{2}+B_{3} z^{3}+\ldots\left(B_{1}>0\right) .
$$

Using this $\phi$, Ma and Minda [12] considered the classes

$$
S(\phi)=\left\{f \in A: \frac{z f^{\prime}(z)}{f(z)} \prec \phi(z), z \in U\right\}
$$

and

$$
K(\phi)=\left\{f \in A: z f^{\prime}(z) \in S(\phi), z \in U\right\}
$$

Several well-known classes can be obtained by specializing of the function $\phi$, for instance

1. By taking $\phi(z)=\frac{1+A z}{1+B z},-1 \leq B<A \leq 1$, we obtain the classes $S[A, B]$ and $K[A, B]$ of the well-known Janowski starlike and convex functions.

2. If we set $\phi(z)=\frac{1+(1-2 \alpha) z}{1-z}$, we obtain the classes $S^{*}(\alpha)$ and $K(\alpha)$ of starlike and convex functions of order $\alpha(0 \leq \alpha<1)$.

3. The class $S_{L}^{*}:=S(\sqrt{1+z})$ was considered by Sokol and Stankieicz [13], consisting of functions $f$ such that $\frac{z f^{\prime}(z)}{f(z)}$ lies in the region bounded by the right half of the Bernoulli lemniscate given by $\left|w^{2}-1\right|<1$.

4. Taking $\phi(z)=\left(\frac{1+z}{1-z}\right)^{\delta}(0<\delta \leq 1)$ yields the classes of strongly starlike and convex functions. 
5. The function class $S_{\nabla}^{*}:=S\left(z+\sqrt{1+z^{2}}\right)$ was considered by Raina and Sokol [14], consisting of normalized starlike functions $f$ satisfying the inequality

$$
\left|\left\{\frac{z f^{\prime}(z)}{f(z)}\right\}^{2}-1\right|<2\left|\frac{z f^{\prime}(z)}{f(z)}\right| \text {. }
$$

6. Kanas et al. [15] considered the family of analytic functions $S\left(\frac{1}{(1-z)^{s}}\right)$ and $K\left(\frac{1}{(1-z)^{s}}\right)$ with the property that $\frac{z f^{\prime}(z)}{f(z)}$ and $1+\frac{z f^{\prime \prime}(z)}{f^{\prime}(z)}$ lie in adomain bounded by the righ branch of a hyperbola $\rho=\rho(s)=\left(2 \cos \frac{\varphi}{s}\right)^{-1}\left(0<s \leq 1,|\varphi|<\frac{\pi s}{2}\right)$.

7. The function class $S_{e}^{*}:=S\left(e^{z}\right)$ was introduced and studied by Mendiratta et al. [16]. The exponential function $\phi(z)=e^{z}$ has positive real part in $U$, maps $U$ onto a domain $\phi(U):=\{w \in C:|\log w|<1\}$ is symmetric with respect to the real axis and starlike with respect to 1 and $\phi^{\prime}(0)>0$.

8. The classes $S\left(\frac{2}{1+e^{-z}}\right)$ and $K\left(\frac{2}{1+e^{-z}}\right)$ were introduced and studied by Goel and Kumar [17]. The modified sigmoid function $\phi(z)=\frac{2}{1+e^{-z}}$ maps $U$ onto a domain $S_{G}:=\{w \in C:|\log (w /(2-w))|<1\}$, which is symmetric about the real axis. Moreover, $G$ is a convex and hence starlike function with respect to $G(0)=1$.

An interesting families of the domains that are bounded by a conic sections were introduced and studied by Shams et al. [18], they introduced the class $S D(\alpha, \beta)$ of $\beta$ uniformly starlike functions of order $\alpha(0 \leq \alpha<1)$ in $U$ consisting of functions $f \in A$ which satisfy the following inequality

$$
\Re\left\{\frac{z f^{\prime}(z)}{f(z)}-\alpha\right\}>\beta\left|\frac{z f^{\prime}(z)}{f(z)}-1\right| \quad(\beta \geq 0 ; 0 \leq \alpha<1 ; z \in U) .
$$

and class $K D(\alpha, \beta)$ of $\beta$-uniformly convex of order $\alpha(0 \leq \alpha<1)$, defined by

$$
f \in K D(\alpha, \beta) \Leftrightarrow z f^{\prime}(z) \in S D(\alpha, \beta) .
$$

Since $\operatorname{Re} w>\alpha|w-1|+\gamma$ if and only if $\operatorname{Re}\left\{w\left(1+\alpha e^{i \theta}\right)-\alpha e^{i \theta}\right\}>\gamma$ (see [19]), then the condition (5) is equivalent to

$$
\Re\left\{\left(1+\beta e^{i \theta}\right) \frac{z f^{\prime}(z)}{f(z)}-\beta e^{i \theta}\right\}>\alpha .
$$

Motivated by the classes $S D(\alpha, \beta)$ and $K D(\alpha, \beta)$ we now introduce and investigate the following subclasses of $A$, and obtain some interesting results.

Definition 1. A function $f(z) \in A$ is said to be in the class $M(\lambda, \beta, \gamma, \phi)$ if it satisfies

$$
\left(1+\beta e^{i \gamma}\right) \frac{z f^{\prime}(z)+\lambda z^{2} f^{\prime \prime}(z)}{(1-\lambda) f(z)+\lambda z f^{\prime}(z)}-\beta e^{i \gamma} \prec \phi(z) \quad(z \in U),
$$

where $\beta \geq 0,0 \leq \lambda \leq 1$ and $-\pi \leq \gamma<\pi$.

We note that:

1. The class $M(0,0, \gamma, \phi)=S(\phi)$ and the class $M(1,0, \gamma, \phi)=K(\phi)$.

2. The class $M\left(0, \beta, \gamma, \frac{1+(1-2 \alpha) z}{1-z}\right)=S D(\alpha, \beta)$ and the class $M\left(1, \beta, \gamma, \frac{1+(1-2 \alpha) z}{1-z}\right)=K D(\alpha, \beta)$.

3. The class $M\left(\lambda, 0, \gamma, \frac{1+(1-2 \alpha) z}{1-z}\right)$ was introduced and studied by Aouf et al. [20]. 
Definition 2. A function $f(z) \in A$ is said to be in the class $S(\lambda, \beta, \gamma, \phi)$ if it satisfies

$$
\left(1+\beta e^{i \gamma}\right)\left[(1-\lambda) \frac{z f^{\prime}(z)}{f(z)}+\lambda\left(1+\frac{z f^{\prime \prime}(z)}{f^{\prime}(z)}\right)\right]-\beta e^{i \gamma} \prec \phi(z) \quad(z \in U),
$$

where $\beta \geq 0,0 \leq \lambda \leq 1$ and $-\pi \leq \gamma<\pi$

We note that:

1. The class $S(0,0, \gamma, \phi)=S(\phi)$ and $S(1,0, \gamma, \phi)=K(\phi)$.

2. $M\left(1, \beta, \gamma, \frac{1+(1-2 \alpha) z}{1-z}\right)=S D(\alpha, \beta)$ and $S\left(0, \beta, \gamma, \frac{1+(1-2 \alpha) z}{1-z}\right)=K D(\alpha, \beta)$.

A single-valued function $f$ analytic in a domain $D \subset C$ is said to be univalent there if it never take the same value twice; that is, if $f\left(z_{1}\right) \neq f\left(z_{2}\right)$ for all points $z_{1}$ and $z_{2}$ in $D$ with $z_{1} \neq z_{2}$ (see [8], p. 26). A function $f \in A$ is said to be bi-univalent in $U$ if $f$ and its inverse map $f^{-1}$ are univalent in $U$. Let $\sigma$ denote the class of bi-univalent functions in $U$ given by (1). The class of analytic bi-univalent functions was first introduced and studied by Lewin [21] and showed that $\left|a_{2}\right|<1.51$. Recently, many authors found non-sharp estimates on the first two Taylor-Maclaurin coefficients $\left|a_{2}\right|$ and $\left|a_{3}\right|$ for various subclasses of bi-univalent functions, see for example, ([22-43]). For other related topics see also, ([44-47]).

Definition 3. A function $f \in \sigma$ given by (1) is said to be in the class $M_{\sigma}(\lambda, \beta, \gamma, \phi)$ if both $f$ and its inverse map $g=f^{-1}$ are in $M(\lambda, \beta, \gamma, \phi)$.

We note that:

1. The class $M_{\sigma}(0,1, \gamma, \phi)=\Re_{\sigma}(\phi)$ was introduced and studied by Darwish et al. [48].

2. The class $M_{\sigma}\left(0,0, \gamma, \frac{1+A z}{1+B z}\right)=S[A, B]$ was introduced and studied by Hamidi and Jahangiri [49].

Definition 4. A function $f \in \sigma$ given by (1) is said to be in the class $S_{\sigma}(\lambda, \beta, \gamma, \phi)$ if both $f$ and its inverse map $g=f^{-1}$ are in $S(\lambda, \beta, \gamma, \phi)$.

We note that:

1. The class $S_{\sigma}(0,1, \gamma, \phi)=\Re_{\sigma}(\phi)$.

2. The class $S_{\sigma}\left(0,0, \gamma, \frac{1+A z}{1+B z}\right)=S[A, B]$.

3. The class $S_{\sigma}(\lambda, 0, \gamma, \phi)=M_{q}^{\sigma}(\lambda, \phi)$ was introduced and studied by Goyal and Kumar [50], see also Zireh et al. [51].

In this paper, we use the Faber polynomial expansions to obtain bounds for the general coefficients $\left|a_{n}\right|$ of bi-univalent functions in $M_{\sigma}(\lambda, \beta, \gamma, \phi)$ and $S_{\sigma}(\lambda, \beta, \gamma, \phi)$ as well as we provide estimates for the initial coefficients of these functions.

\section{Coefficient Estimates for the Class $M_{\sigma}(p, \lambda, \tau, \phi)$}

Theorem 1. Let the function $f \in \sigma$ given by (1) be in the class $M_{\sigma}(\lambda, \beta, \gamma, \phi)$. If $a_{k}=0$ for $2 \leq k \leq n-1$, then

$$
\left|a_{n}\right| \leq \frac{B_{1}}{(n-1)[1+\lambda(n-1)]\left|1+\beta e^{i \gamma}\right|}, \quad n \geq 3 .
$$

Proof. If we set $F(z):=(1-\lambda) f(z)+\lambda z f^{\prime}(z)=z+\sum_{n=2}^{\infty}[1+\lambda(n-1)] a_{n} z^{n}:=z+\sum_{n=2}^{\infty} \delta_{n} z^{n}$, then

$$
f \in M(\lambda, \beta, \gamma, \phi) \Leftrightarrow\left(1+\beta e^{i \gamma}\right) \frac{z F^{\prime}(z)}{F(z)}-\beta e^{i \gamma} \prec \phi(z) .
$$


Since, both functions $f$ and its inverse map $g=f^{-1}$ are in $M(\lambda, \beta, \gamma, \phi)$, by the definition of subordination, there are analytic functions $u, v: U \rightarrow U$ with $u(0)=v(0)=$ $0,|u(z)|<1$ and $|v(z)|<1$, such that

$$
\left(1+\beta e^{i \gamma}\right) \frac{z F^{\prime}(z)}{F(z)}-\beta e^{i \gamma}=\phi(u(z)) \quad(z \in U)
$$

and

$$
\left(1+\beta e^{i \gamma}\right) \frac{w G^{\prime}(w)}{G(w)}-\beta e^{i \gamma}=\phi(v(w)) \quad(z \in U)
$$

where $G(z):=(1-\lambda) g(z)+\lambda z g^{\prime}(z)=z+\sum_{n=2}^{\infty}[1+\lambda(n-1)] d_{n} z^{n}:=z+\sum_{n=2}^{\infty} \zeta_{n} z^{n}$ and $d_{n}=\frac{1}{n} K_{n-1}^{-n}\left(a_{2}, a_{3}, \ldots, a_{n}\right)$. Define the functions $u(z)$ and $v(z)$ by

$$
u(z)=\sum_{n=1}^{\infty} b_{n} z^{n}, \quad v(z)=\sum_{n=1}^{\infty} c_{n} z^{n} \quad(z \in U) .
$$

It is well known that (see Duren [8], p. 265)

$$
\left|b_{n}\right| \leq 1,\left|c_{n}\right| \leq 1 \quad n=2,3, \ldots
$$

By a simple calculation, we have

$$
\begin{aligned}
\phi(u(z)) & =1-B_{1} \sum_{n=1}^{\infty} K_{n}^{-1}\left(b_{1}, b_{2}, \ldots, b_{n}, B_{1}, B_{1}, B_{2}, B_{3}, \ldots, B_{n}\right) z^{n} \\
& =1+B_{1} b_{1} z+\left(B_{1} b_{2}+B_{2} b_{1}^{2}\right) z^{2}+\ldots(z \in U),
\end{aligned}
$$

and

$$
\begin{aligned}
\phi(v(\omega)) & =1-B_{1} \sum_{n=1}^{\infty} K_{n}^{-1}\left(c_{1}, c_{2}, \ldots, c_{n}, B_{1}, B_{2}, B_{3}, \ldots, B_{n}\right) w^{n} \\
& =1+B_{1} c_{1} \omega+\left(B_{1} c_{2}+B_{2} c_{1}^{2}\right) \omega^{2}+\ldots(\omega \in U),
\end{aligned}
$$

In general (see [52], p. 649), the coefficients $K_{n}^{p}\left(k_{1}, k_{2}, \ldots, k_{n}, B_{1}, B_{2}, B_{3}, \ldots, B_{n}\right)$ are given by

$$
\begin{aligned}
& K_{n}^{p}\left(k_{1}, k_{2}, \ldots, k_{n}, B_{1}, B_{2}, B_{3}, \ldots, B_{n}\right) \\
& =\frac{p !}{(p-n) ! n !} k_{1}^{n} \frac{(-1)^{n+1} B_{n}}{B_{1}}+\frac{p !}{(p-n+1) !(n-2) !} k_{1}^{n-2} k_{2} \frac{(-1)^{n} B_{n-1}}{B_{1}} \\
& +\frac{p !}{(p-n+2) !(n-3) !} k_{1}^{n-3} k_{3} \frac{(-1)^{n-1} B_{n-2}}{B_{1}} \\
& +\frac{p !}{(p-n+3) !(n-4) !} k_{1}^{n-4}\left[k_{4} \frac{(-1)^{n-2} B_{n-3}}{B_{1}}+\frac{p-n+3}{2} k_{2}^{2} k_{3} \frac{(-1)^{n-1} B_{n-2}}{B_{1}}\right] \\
& +\sum_{j \geq 5}^{\infty} k_{1}^{n-j} X_{j},
\end{aligned}
$$

where $X_{j}$ is a homogeneous polynomial of degree $j$ in the variables $k_{2}, \ldots, k_{n}$.

Using the Faber polynomial expansion (2) yield the following identities

$$
\left(1+\beta e^{i \gamma}\right) \frac{z F^{\prime}(z)}{F(z)}-\beta e^{i \gamma}=\left(1+\beta e^{i \gamma}\right)\left[1-\sum_{j=2}^{\infty} F_{j-1}\left(\delta_{2}, \delta_{3}, \ldots, \delta_{j}\right) z^{j-1}\right]-\beta e^{i \gamma},
$$


and

$$
\left(1+\beta e^{i \gamma}\right) \frac{w G^{\prime}(w)}{G(w)}-\beta e^{i \gamma}=\left(1+\beta e^{i \gamma}\right)\left[1-\sum_{j=2}^{\infty} F_{j-1}\left(\zeta_{2}, \zeta_{3}, \ldots, \zeta_{j}\right) w^{j-1}\right]-\beta e^{i \gamma} .
$$

Comparing the corresponding coefficients of (10) and (12) yields

$$
\left(1+\beta e^{i \gamma}\right) F_{n-1}\left(\delta_{2}, \delta_{3}, \ldots, \delta_{n}\right)=B_{1} K_{n-1}^{-1}\left(b_{1}, b_{2}, \ldots, b_{n-1}, B_{1}, B_{2}, B_{3}, \ldots, B_{n-1}\right)
$$

and similarly, from (11) and (13), we have

$$
\left(1+\beta e^{i \gamma}\right) F_{n-1}\left(\zeta_{2}, \zeta_{3}, \ldots, \zeta_{n}\right)=B_{1} K_{n-1}^{-1}\left(c_{1}, c_{2}, \ldots, c_{n-1}, B_{1}, B_{2}, B_{3}, \ldots, B_{n-1}\right) .
$$

Since $a_{k}=0$ for $2 \leq k \leq n-1$, by substituting $\delta_{n}=[1+\lambda(n-1)] a_{n}, \zeta_{n}=[1+\lambda(n-$ 1)] $d_{n}$ and $d_{n}=-a_{n}$ in (14) and (15), we have

$$
\left(1+\beta e^{i \gamma}\right)(n-1)[1+\lambda(n-1)] a_{n}=B_{1} b_{n-1}
$$

and

$$
-\left(1+\beta e^{i \gamma}\right)(n-1)[1+\lambda(n-1)] a_{n}=B_{1} c_{n-1} .
$$

By using (9), we conclude that

$$
\left|a_{n}\right| \leq \frac{B_{1}}{\left|1+\beta e^{i \gamma}\right|(n-1)[1+\lambda(n-1)]},
$$

this completes the proof.

To prove our next theorem, we shall need the following lemma .

Lemma 2. Ref. [52] Let the function $\Phi(z)=\sum_{n=1}^{\infty} \Phi_{n} z^{n}$ be a Schwarz function with $|\Phi(z)|<1$, $z \in U$. Then for $-\infty<\rho<\infty$.

$$
\left|\Phi_{2}+\rho \Phi_{1}^{2}\right| \leq \begin{cases}1-(1-\rho)\left|\Phi_{1}^{2}\right| & \rho>0 \\ 1-(1+\rho)\left|\Phi_{1}^{2}\right| & \rho \leq 0\end{cases}
$$

Theorem 2. Let the function $f \in \sigma$ given by (1) be in the class $M_{\sigma}(\lambda, \beta, \gamma, \phi)$, then

$$
\left|a_{2}\right| \leq\left\{\begin{array}{cc}
\frac{B_{1} \sqrt{B_{1}}}{\sqrt{\left|1+\beta e^{i \gamma}\right|\left(1+2 \lambda-\lambda^{2}\right) B_{1}^{2}+\left|1+\beta e^{i \gamma}\right|^{2}(1+\lambda)^{2}\left(B_{1}+B_{2}\right)}} & \left(B_{2} \leq 0, B_{1}+B_{2} \geq 0\right) \\
\frac{B_{1} \sqrt{B_{1}}}{\sqrt{\left|1+\beta e^{i \gamma}\right|\left(1+2 \lambda-\lambda^{2}\right) B_{1}^{2}+\left|1+\beta e^{i \gamma}\right|^{2}(1+\lambda)^{2}\left(B_{1}-B_{2}\right)}} & \left(B_{2}>0, B_{1}-B_{2} \geq 0\right)
\end{array}\right.
$$

and

$$
\left|a_{3}-a_{2}^{2}\right| \leq \begin{cases}\frac{B_{1}}{2(1+2 \lambda)\left|1+\beta e^{i \gamma}\right|} & \left(B_{1} \geq\left|B_{2}\right|\right) \\ \frac{\left|B_{2}\right|}{2(1+2 \lambda)\left|1+\beta e^{i \gamma}\right|} & \left(B_{1}<\left|B_{2}\right|\right) .\end{cases}
$$

Proof. Replacing $n=2$ and 3 in (14) and (15), respectively, we find that

$$
\begin{gathered}
\left(1+\beta e^{i \gamma}\right)(1+\lambda) a_{2}=B_{1} b_{1}, \\
\left(1+\beta e^{i \gamma}\right)\left[2(1+2 \lambda) a_{3}-(1+\lambda)^{2} a_{2}^{2}\right]=\left[B_{1} b_{2}+B_{2} b_{1}^{2}\right], \\
\left(-1+\beta e^{i \gamma}\right)(1+\lambda) a_{2}=B_{1} c_{1}, \\
\left(1+\beta e^{i \gamma}\right)\left\{-2(1+2 \lambda) a_{3}+\left[4(1+2 \lambda)-(1+\lambda)^{2}\right] a_{2}^{2}\right\}=\left[B_{1} c_{2}+B_{2} c_{1}^{2}\right] .
\end{gathered}
$$


It follows from (18) and (20) that

$$
b_{1}=-c_{1}
$$

Adding (19) to (21) implies

$$
2\left(1+\beta e^{i \gamma}\right)\left[2(1+2 \lambda)-(1+\lambda)^{2}\right] a_{2}^{2}=B_{1}\left(b_{2}+c_{2}\right)+B_{2}\left(b_{1}^{2}+c_{1}^{2}\right) .
$$

Taking the absolute values of both sides of the above equation, we get

$$
\left|a_{2}\right|^{2} \leq \frac{B_{1}}{2\left|1+\beta e^{i \gamma}\right|\left(1+2 \lambda-\lambda^{2}\right)}\left(\left|b_{2}+\frac{B_{2}}{B_{1}} b_{1}^{2}\right|+\left|c_{2}+\frac{B_{2}}{B_{1}} c_{1}^{2}\right|\right) .
$$

Case 1. Let $B_{2} \leq 0$. Applying Lemma 2 with $\rho=\frac{B_{2}}{B_{1}} \leq 0$ and using (22) we obtain

$$
\left|a_{2}\right|^{2} \leq \frac{B_{1}}{\left|1+\beta e^{i \gamma}\right|\left(1+2 \lambda-\lambda^{2}\right)}\left(1-\left[\frac{B_{1}+B_{2}}{B_{1}}\right]\left|b_{1}\right|^{2}\right) .
$$

If $B_{1}+B_{2} \geq 0$, then (18) yields

$$
\left|a_{2}\right| \leq \frac{B_{1} \sqrt{B_{1}}}{\sqrt{\left|1+\beta e^{i \gamma}\right|\left(1+2 \lambda-\lambda^{2}\right) B_{1}^{2}+\left|1+\beta e^{i \gamma}\right|^{2}(1+\lambda)^{2}\left(B_{1}+B_{2}\right)}} .
$$

Case 2. Let $B_{2}>0$. Applying Lemma 2 with $\rho=\frac{B_{2}}{B_{1}}>0$ and using (22), we obtain

$$
\left|a_{2}^{2}\right| \leq \frac{B_{1}}{\left|1+\beta e^{i \gamma}\right|\left[2(1+2 \lambda)-(1+\lambda)^{2}\right]}\left(1-\left[\frac{B_{1}-B_{2}}{B_{1}}\right]\left|b_{1}\right|^{2}\right) .
$$

If $B_{1}-B_{2} \geq 0$, then (18) gives

$$
\left|a_{2}\right| \leq \frac{B_{1} \sqrt{B_{1}}}{\sqrt{\left|1+\beta e^{i \gamma}\right|\left[1+2 \lambda-\lambda^{2}\right] B_{1}^{2}+\left|1+\beta e^{i \gamma}\right|^{2}(1+\lambda)^{2}\left(B_{1}-B_{2}\right)}} .
$$

From (25) and (26) we obtain the desired estimate of $\left|a_{2}\right|$ given by (16). Next, from (19) and (21), we have

$$
\left|a_{3}-a_{2}^{2}\right| \leq \frac{B_{1}}{4(1+2 \lambda)\left|1+\beta e^{i \gamma \mid}\right|}\left(\left|b_{2}+\frac{B_{2}}{B_{1}} b_{1}^{2}\right|+\left|c_{2}+\frac{B_{2}}{B_{1}} c_{1}^{2}\right|\right) .
$$

Let $B_{2} \leq 0$. Applying Lemma 2 for $\rho=\frac{B_{2}}{B_{1}} \leq 0$, we get

$$
\left|a_{3}-a_{2}^{2}\right| \leq \frac{B_{1}}{4(1+2 \lambda)\left|1+\beta e^{i \gamma}\right|}\left(\left[1-\frac{B_{1}+B_{2}}{B_{1}}\left|b_{1}\right|^{2}\right]+\left[1-\frac{B_{1}+B_{2}}{B_{1}}\left|c_{1}\right|^{2}\right]\right) .
$$

If $B_{1}+B_{2} \geq 0$, then (28) gives

$$
\left|a_{3}-a_{2}^{2}\right| \leq \frac{B_{1}}{2(1+2 \lambda)\left|1+\beta e^{i \gamma}\right|} .
$$

If $B_{1}+B_{2}<0$, then (9) and (28) lead to

$$
\left|a_{3}-a_{2}^{2}\right| \leq \frac{B_{1}}{2(1+2 \lambda)\left|1+\beta e^{i \gamma \mid}\right|}\left[1-\frac{B_{1}+B_{2}}{B_{1}}\right]=-\frac{B_{2}}{2(1+2 \lambda)\left|1+\beta e^{i \gamma}\right|} .
$$


Let $B_{2}>0$. Applying Lemma 2 for $\rho=\frac{B_{2}}{B_{1}}>0$, (27) gives

$$
\left|a_{3}-a_{2}^{2}\right| \leq \frac{B_{1}}{4(1+2 \lambda)\left|1+\beta e^{i \gamma}\right|}\left(\left[1-\frac{B_{1}-B_{2}}{B_{1}}\left|b_{1}\right|^{2}\right]+\left[1-\frac{B_{1}-B_{2}}{B_{1}}\left|c_{1}\right|^{2}\right]\right) .
$$

If $B_{1}-B_{2} \geq 0$, then (29) gives

$$
\left|a_{3}-a_{2}^{2}\right| \leq \frac{B_{1}}{2(1+2 \lambda)\left|1+\beta e^{i \gamma}\right|} .
$$

If $B_{1}-B_{2}<0$, then from (9) and (29) we have

$$
\left|a_{3}-a_{2}^{2}\right| \leq \frac{B_{1}}{2(1+2 \lambda)\left|1+\beta e^{i \gamma}\right|}\left[1-\frac{B_{1}-B_{2}}{B_{1}}\right]=\frac{B_{2}}{2(1+2 \lambda)\left|1+\beta e^{i \gamma}\right|} .
$$

Which is the second part of assertion (17). This completes the proof of Theorem 2.

Remark 1. If we take $\beta=0$ in Theorem 2 we obtain that the bounds on $\left|a_{3}-a_{2}^{2}\right|$ given by Deniz et al. [52] when $\gamma=1$.

If we set

$$
\phi(z)=\frac{1+A z}{1+B z}=1+(A-B) z-B(A-B) z^{2}+B^{2}(A-B) z^{3} \cdots
$$

in Definition 3 of the bi-univalent function class $M_{\sigma}(\lambda, \beta, \gamma, \phi)$, we obtain a new class $M_{\sigma}(\lambda, \beta, \gamma, A, B)$ given by Definition 5 below.

Definition 5. A function $f \in \sigma$ given by (1) is said to be in the class $M_{\sigma}(\lambda, \beta, \gamma, A, B),-1 \leq$ $B<A \leq 1$, if the following conditions are satisfied:

$$
\left(1+\beta e^{i \gamma}\right) \frac{z f^{\prime}(z)+\lambda z^{2} f^{\prime \prime}(z)}{(1-\lambda) f(z)+\lambda z f^{\prime}(z)}-\beta e^{i \gamma} \prec \frac{1+A z}{1+B z}(z \in U)
$$

and

$$
\left(1+\beta e^{i \gamma}\right) \frac{z g^{\prime}(\omega)+\lambda z^{2} g^{\prime \prime}(\omega)}{(1-\lambda) g(\omega)+\lambda z g^{\prime}(\omega)}-\beta e^{i \gamma} \prec \frac{1+A \omega}{1+B \omega}(\omega \in U),
$$

where $g=f^{-1}$.

Using the parameter setting of Definition 5 in Theorems 1 and 2, respectively, we get the following corollaries.

Corollary 1. Let the function $f \in M_{\sigma}(\lambda, \beta, \gamma, A, B)$ be given by (1). If $a_{k}=0$ for $2 \leq k \leq n-1$, then

$$
\left|a_{n}\right| \leq \frac{(A-B)}{(n-1)[1+\lambda(n-1)]\left|1+\beta e^{i \gamma}\right|}, \quad n \geq 3 .
$$

Corollary 2. If the function $f \in \sigma$ given by (1) be in the class $M_{\sigma}(\lambda, \beta, \gamma, A, B)$, then

$$
\left|a_{2}\right| \leq\left\{\begin{array}{c}
\frac{(A-B)}{\sqrt{\left|1+\beta e^{i \gamma}\right|\left[1+2 \lambda-\lambda^{2}\right](A-B)+\left|1+\beta e^{i \gamma}\right|^{2}(1+\lambda)^{2}(1-B)}}(B \geq 0) \\
\frac{(A-B)}{\sqrt{\left|1+\beta e^{i \gamma}\right|\left[1+2 \lambda-\lambda^{2}\right](A-B)+\left|1+\beta e^{i \gamma}\right|^{2}(1+\lambda)^{2}(1+B)}}(-1 \leq B<0)
\end{array}\right.
$$

and

$$
\left|a_{3}-a_{2}^{2}\right| \leq \frac{A-B}{2(1+2 \lambda)\left|1+\beta e^{i \gamma}\right|} .
$$


If we set

$$
\phi(z)=\left(\frac{1+z}{1-z}\right)^{\delta}=1+2 \delta z+2 \delta^{2} z^{2}+\ldots(0<\delta \leq 1, z \in U)
$$

in Definition 3 of the bi-univalent function class $M_{\sigma}(\lambda, \beta, \gamma, \phi)$, we obtain a new class $M_{\sigma}(\lambda, \beta, \gamma, \delta)$ given by Definition 6 below.

Definition 6. Let $0<\delta \leq 1$. A function $f \in \sigma$ given by (1) is said to be in the class $M_{\sigma}(\lambda, \beta, \gamma, \delta)$, if the following conditions are satisfied:

$$
\left|\arg \left(\left(1+\beta e^{i \gamma}\right) \frac{z f^{\prime}(z)+\lambda z^{2} f^{\prime \prime}(z)}{(1-\lambda) f(z)+\lambda z f^{\prime}(z)}-\beta e^{i \gamma}\right)\right| \leq \frac{\pi}{2} \delta(z \in U)
$$

and

$$
\left|\arg \left(\left(1+\beta e^{i \gamma}\right) \frac{z g^{\prime}(\omega)+\lambda z^{2} g^{\prime \prime}(\omega)}{(1-\lambda) g(\omega)+\lambda z g^{\prime}(\omega)}-\beta e^{i \gamma}\right)\right| \leq \frac{\pi}{2} \delta(\omega \in U),
$$

where $g=f^{-1}$.

Using the parameter setting of Definition 6 in Theorems 1 and 2, respectively, we get the following corollaries.

Corollary 3. Let the function $f \in M_{\sigma}(\lambda, \beta, \gamma, \delta)$ be given by (1). If $a_{k}=0$ for $2 \leq k \leq n-1$, then

$$
\left|a_{n}\right| \leq \frac{2 \delta}{(n-1)[1+\lambda(n-1)]\left|1+\beta e^{i \gamma}\right|}, \quad n \geq 3 .
$$

Corollary 4. Let $0<\delta \leq 1$. If the function $f \in \sigma$ given by (1) be in the class $M_{\sigma}(\lambda, \beta, \gamma, \delta)$, then

$$
\left|a_{2}\right| \leq \frac{2 \delta}{\sqrt{\left|1+\beta e^{i \gamma}\right|\left[1+2 \lambda-\lambda^{2}\right] 2 \delta+\left|1+\beta e^{i \gamma}\right|^{2}(1+\lambda)^{2}(1-\delta)}}
$$

and

$$
\left|a_{3}-a_{2}^{2}\right| \leq \frac{\delta}{(1+2 \lambda)\left|1+\beta e^{i \gamma}\right|}
$$

If we set

$$
\phi(z)=\frac{1+(1-2 v) z}{1-z}=1+2(1-v) z+2(1-v) z^{2}+\ldots(0 \leq v<1, z \in U)
$$

in Definition 3 of the bi-univalent function class $M_{\sigma}(\lambda, \beta, \gamma, \phi)$, we obtain a new class $M_{\sigma}^{v}(\lambda, \beta, \gamma)$ given by Definition 7 below.

Definition 7. Let $0 \leq v<1$. A function $f \in \sigma$ given by (1) is said to be in the class $M_{\sigma}^{v}(\lambda, \beta, \gamma)$ if the following conditions hold true:

$$
\Re\left(\left(1+\beta e^{i \gamma}\right) \frac{z f^{\prime}(z)+\lambda z^{2} f^{\prime \prime}(z)}{(1-\lambda) f(z)+\lambda z f^{\prime}(z)}-\beta e^{i \gamma}\right)>v(z \in U)
$$

and

$$
\Re\left(\left(1+\beta e^{i \gamma}\right) \frac{z g^{\prime}(\omega)+\lambda z^{2} g^{\prime \prime}(\omega)}{(1-\lambda) g(\omega)+\lambda z g^{\prime}(\omega)}-\beta e^{i \gamma}\right)>v(\omega \in U),
$$

where $g=f^{-1}$. 
Using the parameter setting of Definition 7 in Theorems 1 and 2, respectively, we get the following corollaries.

Corollary 5. Let the function $f \in M_{\sigma}^{v}(\lambda, \beta, \gamma)$ be given by (1). If $a_{k}=0$ for $2 \leq k \leq n-1$, then

$$
\left|a_{n}\right| \leq \frac{2(1-v)}{(n-1)[1+\lambda(n-1)]\left|1+\beta e^{i \gamma}\right|}, \quad n \geq 3 .
$$

Corollary 6. Let the function $f \in M_{\sigma}^{v}(\lambda, \beta, \gamma)$ be given by (1). Then

$$
\left|a_{2}\right| \leq \sqrt{\frac{2(1-v)}{\left(1+2 \lambda-\lambda^{2}\right)\left|1+\beta e^{i \gamma}\right|}}
$$

and

$$
\left|a_{3}-a_{2}^{2}\right| \leq \frac{(1-v)}{(1+2 \lambda)\left|1+\beta e^{i \gamma}\right|}
$$

If we set

$$
\phi(z)=\sqrt{1+z}=1+\frac{1}{2} z-\frac{1}{8} z^{2}+\ldots(z \in U)
$$

in Definition 3 of the bi-univalent function class $M_{\sigma}(\lambda, \beta, \gamma, \phi)$, we obtain a new class $M_{L \sigma}(\lambda, \beta, \gamma)$ given by Definition 8 below.

Definition 8. A function $f \in \sigma$ given by (1) is said to be in the class $M_{L \sigma}(\lambda, \beta, \gamma)$, if the following conditions are satisfied:

$$
\left|\left(\left(1+\beta e^{i \gamma}\right) \frac{z f^{\prime}(z)+\lambda z^{2} f^{\prime \prime}(z)}{(1-\lambda) f(z)+\lambda z f^{\prime}(z)}-\beta e^{i \gamma}\right)^{2}-1\right|<1(z \in U)
$$

and

$$
\left|\left(\left(1+\beta e^{i \gamma}\right) \frac{z g^{\prime}(\omega)+\lambda z^{2} g^{\prime \prime}(\omega)}{(1-\lambda) g(\omega)+\lambda z g^{\prime}(\omega)}-\beta e^{i \gamma}\right)^{2}-1\right|<1(\omega \in U),
$$

where $g=f^{-1}$.

Using the parameter setting of Definition 8 in Theorems 1 and 2, respectively, we get the following corollaries.

Corollary 7. Let the function $f \in M_{L \sigma}(\lambda, \beta, \gamma)$ be given by (1). If $a_{k}=0$ for $2 \leq k \leq n-1$, then

$$
\left|a_{n}\right| \leq \frac{1}{2(n-1)[1+\lambda(n-1)]\left|1+\beta e^{i \gamma}\right|}, \quad n \geq 3 .
$$

Corollary 8. If the function $f \in \sigma$ given by (1) be in the class $M_{L \sigma}(\lambda, \beta, \gamma)$, then

$$
\left|a_{2}\right| \leq \frac{1}{\sqrt{2\left|1+\beta e^{i \gamma}\right|\left[1+2 \lambda-\lambda^{2}\right]+3\left|1+\beta e^{i \gamma}\right|^{2}(1+\lambda)^{2}}}
$$

and

$$
\left|a_{3}-a_{2}^{2}\right| \leq \frac{1}{4(1+2 \lambda)\left|1+\beta e^{i \gamma}\right|} .
$$

If we set

$$
\phi(z)=z+\sqrt{1+z^{2}}=1+z+\frac{1}{2} z^{2}-\frac{1}{8} z^{4} \ldots(z \in U),
$$


in Definition 3 of the bi-univalent function class $M_{\sigma}(\lambda, \beta, \gamma, \phi)$, we obtain a new class $M_{\sigma}^{\Delta}(\lambda, \beta, \gamma)$ given by Definition 9 below.

Definition 9. A function $f \in \sigma$ given by (1) is said to be in the class $M_{\sigma}^{\Delta}(\lambda, \beta, \gamma)$ if the following conditions are satisfied:

$$
\begin{aligned}
& \left|\left(\left(1+\beta e^{i \gamma}\right) \frac{z f^{\prime}(z)+\lambda z^{2} f^{\prime \prime}(z)}{(1-\lambda) f(z)+\lambda z f^{\prime}(z)}-\beta e^{i \gamma}\right)^{2}-1\right| \\
& <2\left|\left(1+\beta e^{i \gamma}\right) \frac{z f^{\prime}(z)+\lambda z^{2} f^{\prime \prime}(z)}{(1-\lambda) f(z)+\lambda z f^{\prime}(z)}-\beta e^{i \gamma}\right|(z \in U)
\end{aligned}
$$

and

$$
\begin{aligned}
& \left|\left(\left(1+\beta e^{i \gamma}\right) \frac{z g^{\prime}(\omega)+\lambda z^{2} g^{\prime \prime}(\omega)}{(1-\lambda) g(\omega)+\lambda z g^{\prime}(\omega)}-\beta e^{i \gamma}\right)^{2}-1\right| \\
& <2\left|\left(1+\beta e^{i \gamma}\right) \frac{z g^{\prime}(\omega)+\lambda z^{2} g^{\prime \prime}(\omega)}{(1-\lambda) g(\omega)+\lambda z g^{\prime}(\omega)}-\beta e^{i \gamma}\right|(\omega \in U)
\end{aligned}
$$

where $g=f^{-1}$.

Using the parameter setting of Definition 9 in Theorems 1 and 2, respectively, we get the following corollaries.

Corollary 9. Let the function $f \in M_{\sigma}^{\Delta}(\lambda, \beta, \gamma)$ be given by (1). If $a_{k}=0$ for $2 \leq k \leq n-1$, then

$$
\left|a_{n}\right| \leq \frac{1}{(n-1)[1+\lambda(n-1)]\left|1+\beta e^{i \gamma}\right|}, \quad n \geq 3 .
$$

Corollary 10. If the function $f \in \sigma$ given by (1) be in the class $M_{\sigma}^{\Delta}(\lambda, \beta, \gamma)$, then

$$
\left|a_{2}\right| \leq \sqrt{\frac{2}{2\left|1+\beta e^{i \gamma}\right|\left[1+2 \lambda-\lambda^{2}\right]+\left|1+\beta e^{i \gamma}\right|^{2}(1+\lambda)^{2}}}
$$

and

$$
\left|a_{3}-a_{2}^{2}\right| \leq \frac{1}{2(1+2 \lambda)\left|1+\beta e^{i \gamma}\right|}
$$

If we set

$$
\begin{aligned}
\phi(z) & =\frac{1}{(1-z)^{s}}=1+s z+\frac{s(s+1)}{2} z^{2}+\frac{s(s+1)(s+2)}{6} z^{3} \ldots \\
& =1+\sum_{n=1}^{\infty} \frac{s(s+1) \ldots(s+n-1)}{n !} z^{n} \quad(z \in U),
\end{aligned}
$$

in Definition 3 of the bi-univalent function class $M_{\sigma}(\lambda, \beta, \gamma, \phi)$, we obtain a new class $M_{\sigma}(\lambda, \beta, \gamma, s)$ given by Definition 10 below.

Definition 10. Let $0<s \leq 1$. A function $f \in \sigma$ given by (1) is said to be in the class $M_{\sigma}(\lambda, \beta, \gamma, s)$, if the following conditions are satisfied:

$$
\left(1+\beta e^{i \gamma}\right) \frac{z f^{\prime}(z)+\lambda z^{2} f^{\prime \prime}(z)}{(1-\lambda) f(z)+\lambda z f^{\prime}(z)}-\beta e^{i \gamma} \prec \frac{1}{(1-z)^{s}}(z \in U)
$$


and

$$
\left(1+\beta e^{i \gamma}\right) \frac{z g^{\prime}(\omega)+\lambda z^{2} g^{\prime \prime}(\omega)}{(1-\lambda) g(\omega)+\lambda z g^{\prime}(\omega)}-\beta e^{i \gamma} \prec \frac{1}{(1-\omega)^{s}}(\omega \in U),
$$

where $g=f^{-1}$.

Using the parameter setting of Definition 10 in Theorems 1 and 2, respectively, we get the following corollaries.

Corollary 11. Let the function $f \in M_{\sigma}(\lambda, \beta, \gamma, s)$ be given by (1). If $a_{k}=0$ for $2 \leq k \leq n-1$, then

$$
\left|a_{n}\right| \leq \frac{s}{(n-1)[1+\lambda(n-1)]\left|1+\beta e^{i \gamma}\right|}, \quad n \geq 3 .
$$

Corollary 12. If the function $f \in \sigma$ given by (1) be in the class $M_{\sigma}(\lambda, \beta, \gamma, s)$, then

$$
\left|a_{2}\right| \leq \frac{\sqrt{2} s}{\sqrt{\left|1+\beta e^{i \gamma}\right|\left[1+2 \lambda-\lambda^{2}\right] 2 s+\left|1+\beta e^{i \gamma}\right|^{2}(1+\lambda)^{2}(1-s)}}
$$

and

$$
\left|a_{3}-a_{2}^{2}\right| \leq \frac{s}{2(1+2 \lambda)\left|1+\beta e^{i \gamma}\right|}
$$

If we set

$$
\phi(z)=e^{z}=1+z+\frac{1}{2} z^{2}+\frac{1}{6} z^{3}+\ldots \quad(z \in U),
$$

in Definition 3 of the bi-univalent function class $M_{\sigma}(\lambda, \beta, \gamma, \phi)$, we obtain a new class $M_{\sigma e}(\lambda, \beta, \gamma)$ given by Definition 11 below.

Definition 11. A function $f \in \sigma$ given by (1) is said to be in the class $M_{\sigma e}(\lambda, \beta, \gamma)$ if the following conditions are satisfied:

$$
\left|\log \left(\left(1+\beta e^{i \gamma}\right) \frac{z f^{\prime}(z)+\lambda z^{2} f^{\prime \prime}(z)}{(1-\lambda) f(z)+\lambda z f^{\prime}(z)}-\beta e^{i \gamma}\right)\right|<1(z \in U)
$$

and

$$
\left|\log \left(\left(1+\beta e^{i \gamma}\right) \frac{z g^{\prime}(\omega)+\lambda z^{2} g^{\prime \prime}(\omega)}{(1-\lambda) g(\omega)+\lambda z g^{\prime}(\omega)}-\beta e^{i \gamma}\right)\right|<1(\omega \in U),
$$

where $g=f^{-1}$.

Using the parameter setting of Definition 11 in Theorems 1 and 2, respectively, we get the following corollaries.

Corollary 13. Let the function $f \in M_{\sigma}(\lambda, \beta, \gamma, s)$, be given by (1). If $a_{k}=0$ for $2 \leq k \leq n-1$, then

$$
\left|a_{n}\right| \leq \frac{1}{(n-1)[1+\lambda(n-1)]\left|1+\beta e^{i \gamma}\right|}, \quad n \geq 3 .
$$

Corollary 14. If the function $f \in \sigma$ given by (1) be in the class $M_{\sigma}(\lambda, \beta, \gamma, s)$, then

$$
\left|a_{2}\right| \leq \sqrt{\frac{2}{2\left|1+\beta e^{i \gamma}\right|\left[1+2 \lambda-\lambda^{2}\right]+\left|1+\beta e^{i \gamma}\right|^{2}(1+\lambda)^{2}}}
$$


and

$$
\left|a_{3}-a_{2}^{2}\right| \leq \frac{1}{2(1+2 \lambda)\left|1+\beta e^{i \gamma}\right|} .
$$

\section{Coefficient Estimates for the Class $S_{\sigma}(\lambda, \beta, \gamma, \phi)$}

Theorem 3. Let the function $f \in \sigma$ given by (1) be in the class $S_{\sigma}(\lambda, \beta, \gamma, \phi)$. If $a_{k}=0$ for $2 \leq k \leq n-1$, then

$$
\left|a_{n}\right| \leq \frac{B_{1}}{\left|1+\beta e^{i \gamma}\right|(n-1)[1+(n-1) \lambda]}, \quad n \geq 3 .
$$

Proof. Since, both functions $f$ and its inverse map $g=f^{-1}$ are in $S_{\sigma}(\lambda, \beta, \gamma, \phi)$, by the definition of subordination, there are analytic functions $u, v: U \rightarrow U$ given by (8) such that

$$
\left(1+\beta e^{i \gamma}\right)\left[(1-\lambda) \frac{z f^{\prime}(z)}{f(z)}+\lambda \frac{\left(z f^{\prime}(z)\right)^{\prime}}{f^{\prime}(z)}\right]-\beta e^{i \gamma}=\phi(u(z))
$$

and

$$
\left(1+\beta e^{i \gamma}\right)\left[(1-\lambda) \frac{w g^{\prime}(w)}{g(w)}+\lambda \frac{\left(w g^{\prime}(w)\right)^{\prime}}{g^{\prime}(w)}\right]-\beta e^{i \gamma}=\phi(v(\omega)) .
$$

Now, from (2), we get that

$$
\begin{aligned}
& \left(1+\beta e^{i \gamma}\right)\left[(1-\lambda) \frac{z f^{\prime}(z)}{f(z)}+\lambda \frac{\left(z f^{\prime}(z)\right)^{\prime}}{f^{\prime}(z)}\right]-\beta e^{i \gamma} \\
& =1-\left(1+\beta e^{i \gamma}\right) \sum_{j=2}^{\infty}\left[(1-\lambda) F_{j-1}\left(a_{2}, a_{3}, \ldots, a_{j}\right)\right. \\
& \left.+\lambda F_{j-1}\left(2 a_{2}, 3 a_{3}, \ldots, j a_{j}\right)\right] z^{j-1}
\end{aligned}
$$

and

$$
\begin{aligned}
& \left(1+\beta e^{i \gamma}\right)\left[(1-\lambda) \frac{w g^{\prime}(w)}{g(w)}+\lambda \frac{\left(w g^{\prime}(w)\right)^{\prime}}{g^{\prime}(w)}\right]-\beta e^{i \gamma} \\
& =1-\left(1+\beta e^{i \gamma}\right) \sum_{j=2}^{\infty}\left[(1-\lambda) F_{j-1}\left(d_{2}, d_{3}, \ldots, d_{j}\right)\right. \\
& \left.+\lambda F_{j-1}\left(2 d_{2}, 3 d_{3}, \ldots, j d_{j}\right)\right] w^{j-1},
\end{aligned}
$$

where $d_{n}=\frac{1}{n} K_{n-1}^{-n}\left(a_{2}, a_{3}, \ldots, a_{n}\right)$. Now, upon comparing the corresponding coefficients in (10) and (30), we get

$$
\begin{aligned}
& \left(1+\beta e^{i \gamma}\right)\left[(1-\lambda) F_{n-1}\left(a_{2}, a_{3}, \ldots, a_{n}\right)+\lambda F_{n-1}\left(2 a_{2}, 3 a_{3}, \ldots, n a_{n}\right)\right] \\
& =B_{1} K_{n-1}^{-1}\left(b_{1}, b_{2}, \ldots, b_{n-1}, B_{1}, B_{1}, B_{2}, B_{3}, \ldots, B_{n-1}\right)
\end{aligned}
$$

and similarly, from (11) and (31), we have

$$
\begin{aligned}
& \left(1+\beta e^{i \gamma}\right)\left[(1-\lambda) F_{n-1}\left(d_{2}, d_{3}, \ldots, d_{n}\right)+\lambda F_{n-1}\left(2 d_{2}, 3 d_{3}, \ldots, n d_{n}\right)\right] \\
& =B_{1} K_{n-1}^{-1}\left(c_{1}, c_{2}, \ldots, c_{n-1}, B_{1}, B_{2}, B_{3}, \ldots, B_{n-1}\right) .
\end{aligned}
$$


Since $a_{k}=0$ for $2 \leq k \leq n-1$, by using $d_{n}=-a_{n}$ and $F_{n-1}\left(a_{2}, a_{3}, \ldots, a_{n}\right)=$ $-(n-1) a_{n}$, we have

$$
\left(1+\beta e^{i \gamma}\right)(n-1)[1+(n-1) \lambda] a_{n}=B_{1} b_{n-1}
$$

and

$$
-\left(1+\beta e^{i \gamma}\right)(n-1)[1+(n-1) \lambda] a_{n}=B_{1} c_{n-1} .
$$

By using (9), we conclude that

$$
\left|a_{n}\right| \leq \frac{B_{1}}{\left|1+\beta e^{i \gamma}\right|(n-1)[1+(n-1) \lambda]} .
$$

Remark 2. If we take $\beta=0$ in Theorem 3, then we have the results which were given by Zireh et al. [51] when $\varphi(z)=1$.

Theorem 4. If the function $f \in \sigma$ given by (1) be in the class $S_{\sigma}(\lambda, \beta, \gamma, \phi)$, then

$$
\left|a_{2}\right| \leq\left\{\begin{array}{cc}
\frac{B_{1} \sqrt{B_{1}}}{\sqrt{\left|1+\beta e^{i \gamma}\right|(1+\lambda) B_{1}^{2}+\left|1+\beta e^{i \gamma}\right|^{2}(1+\lambda)^{2}\left(B_{1}+B_{2}\right)}} & \left(B_{2} \leq 0, B_{1}+B_{2} \geq 0\right) \\
\frac{B_{1} \sqrt{B_{1}}}{\sqrt{\left|1+\beta e^{i \gamma}\right|(1+\lambda) B_{1}^{2}+\left|1+\beta e^{i \gamma}\right|^{2}(1+\lambda)^{2}\left(B_{1}-B_{2}\right)}} & \left(B_{2}>0, B_{1}-B_{2} \geq 0\right)
\end{array},\right.
$$

and

$$
\left|a_{3}-a_{2}^{2}\right| \leq \begin{cases}\frac{B_{1}}{2\left|1+\beta e^{i \gamma}\right|(1+2 \lambda)} & \left(B_{1} \geq\left|B_{2}\right|\right) \\ \frac{\left|B_{2}\right|}{2\left|1+\beta e^{i \gamma}\right|(1+2 \lambda)} & \left(B_{1}<\left|B_{2}\right|\right) .\end{cases}
$$

Proof. Letting $n=2$ and 3 in (32) and (33), respectively, we find that

$$
\begin{gathered}
\left(1+\beta e^{i \gamma}\right)(1+\lambda) a_{2}=B_{1} b_{1}, \\
\left(1+\beta e^{i \gamma}\right)\left[2(1+2 \lambda) a_{3}-(1+3 \lambda) a_{2}^{2}\right]=B_{1} b_{2}+B_{2} b_{1}^{2}, \\
-\left(1+\beta e^{i \gamma}\right)(1+\lambda) a_{2}=B_{1} c_{1}, \\
\left(1+\beta e^{i \gamma}\right)\left\{-2(1+2 \lambda) a_{3}+[4(1+2 \lambda)-(1+3 \lambda)] a_{2}^{2}\right\}=B_{1} c_{2}+B_{2} c_{1}^{2} .
\end{gathered}
$$

Equations (38) and (40) lead to

$$
b_{1}=-c_{1} \text {. }
$$

Adding (39) and (41) yields

$$
2\left(1+\beta e^{i \gamma}\right)(1+\lambda) a_{2}^{2}=B_{1}\left(b_{2}+c_{2}\right)+B_{2}\left(b_{1}^{2}+c_{1}^{2}\right)
$$

or

$$
\left|a_{2}^{2}\right| \leq \frac{B_{1}}{2\left|1+\beta e^{i \gamma}\right|(1+\lambda)}\left(\left|b_{2}+\frac{B_{2}}{B_{1}} b_{1}^{2}\right|+\left|c_{2}+\frac{B_{2}}{B_{1}} c_{1}^{2}\right|\right) .
$$

First, let $B_{2} \leq 0$. Applying Lemma 2 with $\rho=\frac{B_{2}}{B_{1}} \leq 0$ and using (42), we get

$$
\left|a_{2}^{2}\right| \leq \frac{B_{1}}{\left|1+\beta e^{i \gamma}\right|(1+\lambda)}\left(1-\left[\frac{B_{1}+B_{2}}{B_{1}}\right]\left|b_{1}^{2}\right|\right)
$$


If $B_{1}+B_{2} \geq 0$, then (38) yields

$$
\left|a_{2}\right| \leq \frac{B_{1} \sqrt{B_{1}}}{\sqrt{\left|1+\beta e^{i \gamma}\right|(1+\lambda) B_{1}^{2}+\left|1+\beta e^{i \gamma}\right|^{2}(1+\lambda)^{2}\left(B_{1}+B_{2}\right)}}
$$

Similarly, for $B_{2}>0\left(\rho=\frac{B_{2}}{B_{1}}>0, B_{1}-B_{2} \geq 0\right)$, we have

$$
\left|a_{2}\right| \leq \frac{B_{1} \sqrt{B_{1}}}{\sqrt{\left|1+\beta e^{i \gamma}\right|(1+\lambda) B_{1}^{2}+\left|1+\beta e^{i \gamma}\right|^{2}(1+\lambda)^{2}\left(B_{1}-B_{2}\right)}}
$$

From (45) and (46) we obtain the desired estimate of $\left|a_{2}\right|$ given by (36).

Next, in order to find the bound on $\left|a_{3}-a_{2}^{2}\right|$, by subtracting (41) from (39), we have

$$
\left|a_{3}-a_{2}^{2}\right| \leq \frac{B_{1}}{4\left|1+\beta e^{i \gamma}\right|(1+2 \lambda)}\left(\left|b_{2}+\frac{B_{2}}{B_{1}} b_{1}^{2}\right|+\left|c_{2}+\frac{B_{2}}{B_{1}} c_{1}^{2}\right|\right) .
$$

Let $B_{2} \leq 0$. Applying Lemma 2 with $\rho=\frac{B_{2}}{B_{1}} \leq 0$, we get

$$
\left|a_{3}-a_{2}^{2}\right| \leq \frac{B_{1}}{4\left|1+\beta e^{i \gamma}\right|(1+2 \lambda)}\left(\left[1-\frac{B_{1}+B_{2}}{B_{1}}\left|b_{1}\right|^{2}\right]+\left[1-\frac{B_{1}+B_{2}}{B_{1}}\left|c_{1}\right|^{2}\right]\right) .
$$

If $B_{1}+B_{2} \geq 0$, then (48) gives $\left|a_{3}-a_{2}^{2}\right| \leq \frac{B_{1}}{2\left|1+\beta e^{i \gamma}\right|(1+2 \lambda)}$.

If $B_{1}+B_{2}<0$, then (9) and (48) give

$$
\left|a_{3}-a_{2}^{2}\right| \leq \frac{B_{1}}{2\left|1+\beta e^{i \gamma}\right|(1+2 \lambda)}\left[1-\frac{B_{1}+B_{2}}{B_{1}}\right]=-\frac{B_{2}}{2\left|1+\beta e^{i \gamma}\right|(1+2 \lambda)} .
$$

Let $B_{2}>0$. Applying Lemma 2 with $\rho=\frac{B_{2}}{B_{1}}>0$, (47) gives

$$
\left|a_{3}-a_{2}^{2}\right| \leq \frac{B_{1}}{4\left|1+\beta e^{i \gamma}\right|(1+2 \lambda)}\left(\left[1-\frac{B_{1}-B_{2}}{B_{1}}\left|b_{1}\right|^{2}\right]+\left[1-\frac{B_{1}-B_{2}}{B_{1}}\left|c_{1}\right|^{2}\right]\right) .
$$

If $B_{1}-B_{2} \geq 0$, then (49) gives

$$
\left|a_{3}-a_{2}^{2}\right| \leq \frac{B_{1}}{2\left|1+\beta e^{i \gamma}\right|(1+2 \lambda)} .
$$

If $B_{1}-B_{2}<0$, then from (9) and (49) we get

$$
\left|a_{3}-a_{2}^{2}\right| \leq \frac{B_{1}}{2\left|1+\beta e^{i \gamma}\right|(1+2 \lambda)}\left[1-\frac{B_{1}-B_{2}}{B_{1}}\right]=\frac{B_{2}}{2\left|1+\beta e^{i \gamma}\right|(1+2 \lambda)} .
$$

This completes the proof of Theorem 3.

Remark 3. If we set $\beta=0$ in Theorem 4, then we obtain the results of Goyal and Kumar [50] when $\varphi(z)=1$.

If we set $\phi(z)=\left(\frac{1+z}{1-z}\right)^{\delta}(0<\delta \leq 1, z \in U)$ in Definition 4 of the bi-univalent function class $S_{\sigma}(\lambda, \beta, \gamma, \phi)$, we obtain a new class $S_{\sigma}^{\delta}(\lambda, \beta, \gamma)$ given by Definition 12 below. 
Definition 12. Let $0<\delta \leq 1$. A function $f \in \sigma$ given by (1) is said to be in the class $S_{\sigma}^{\delta}(\lambda, \beta, \gamma)$ if the following subordinations hold:

$$
\left|\arg \left(\left(1+\beta e^{i \gamma}\right)\left[(1-\lambda) \frac{z f^{\prime}(z)}{f(z)}+\lambda\left(1+\frac{z f^{\prime \prime}(z)}{f^{\prime}(z)}\right)\right]-\beta e^{i \gamma}\right)\right| \leq \frac{\pi}{2} \delta(z \in U)
$$

and

$$
\left|\arg \left(\left(1+\beta e^{i \gamma}\right)\left[(1-\lambda) \frac{w g^{\prime}(w)}{g(w)}+\lambda\left(1+\frac{w g^{\prime \prime}(w)}{g^{\prime}(w)}\right)\right]-\beta e^{i \gamma}\right)\right| \leq \frac{\pi}{2} \delta(\omega \in U),
$$

where $g=f^{-1}$.

Using the parameter setting of Definition 12 in Theorems 3 and 4, respectively, we get the following corollaries.

Corollary 15. Let the function $f \in S_{\sigma}^{\delta}(\lambda, \beta, \gamma)$ be given by (1). If $a_{k}=0$ for $2 \leq k \leq n-1$, then

$$
\left|a_{n}\right| \leq \frac{2 \delta}{(n-1)[1+\lambda(n-1)]\left|1+\beta e^{i \gamma}\right|}, \quad n \geq 3 .
$$

Corollary 16. Let $0<\gamma \leq 1$. If the function $f \in \sigma$ given by (1) be in the class $S_{\sigma}^{\delta}(\lambda, \beta, \gamma)$, then

$$
\left|a_{2}\right| \leq \frac{2 \delta}{\sqrt{2\left|1+\beta e^{i \gamma}\right|(1+\lambda) \delta+\left|1+\beta e^{i \gamma}\right|^{2}(1+\lambda)^{2}(1-\delta)}}
$$

and

$$
\left|a_{3}-a_{2}^{2}\right| \leq \frac{\delta}{\left|1+\beta e^{i \gamma}\right|(1+2 \lambda)} .
$$

If we set $\phi(z)=\frac{1+(1-2 v) z}{1-z}(0 \leq v<1, z \in U)$ in Definition 4 of the bi-univalent function class $S_{\sigma}(\lambda, \beta, \gamma, \phi)$, we obtain a new class $S_{\sigma}(\lambda, \beta, \gamma, v)$ given by Definition 13 below.

Definition 13. Let $0 \leq v<1$. A function $f \in \sigma$ given by (1) is said to be in the class $S_{\sigma}(\lambda, \beta, \gamma, v)$, if the following conditions are satisfied:

$$
\Re\left(\left(1+\beta e^{i \gamma}\right)\left[(1-\lambda) \frac{z f^{\prime}(z)}{f(z)}+\lambda\left(1+\frac{z f^{\prime \prime}(z)}{f^{\prime}(z)}\right)\right]-\beta e^{i \gamma}\right)>v(z \in U)
$$

and

$$
\Re\left(\left(1+\beta e^{i \gamma}\right)\left[(1-\lambda) \frac{w g^{\prime}(w)}{g(w)}+\lambda\left(1+\frac{w g^{\prime \prime}(w)}{g^{\prime}(w)}\right)\right]-\beta e^{i \gamma}\right)>v(\omega \in U),
$$

where $g=f^{-1}$.

Using the parameter setting of Definition 13 in Theorems 3 and 4, respectively, we get the following corollaries.

Corollary 17. Let the function $f \in S_{\sigma}(\lambda, \beta, \gamma, v)$ be given by (1). If $a_{k}=0$ for $2 \leq k \leq n-1$, then

$$
\left|a_{n}\right| \leq \frac{2(1-v)}{(n-1)[1+\lambda(n-1)]\left|1+\beta e^{i \gamma}\right|}, \quad n \geq 3 .
$$


Corollary 18. Let $0 \leq v<1$. If the function $f \in S_{\sigma}(\lambda, \beta, \gamma, v)$ be of the form (1), then

$$
\left|a_{2}\right| \leq \sqrt{\frac{2(1-v)}{\left|1+\beta e^{i \gamma}\right|(1+\lambda)}}
$$

and

$$
\left|a_{3}-a_{2}^{2}\right| \leq \frac{(1-v)}{\left|1+\beta e^{i \gamma}\right|(1+2 \lambda)}
$$

If we set $\phi(z)=\frac{1+A z}{1+B z}$ in Definition 4 of the bi-univalent function class $S_{\sigma}(\lambda, \beta, \gamma, \phi)$, we obtain a new class $S_{\sigma}(\lambda, \beta, \gamma, A, B)$ given by Definition 14 below.

Definition 14. A function $f \in \sigma$ given by (1) is said to be in the class $S_{\sigma}(\lambda, \beta, \gamma, A, B),-1 \leq$ $B<A \leq 1$, if the following conditions are satisfied:

$$
\left(1+\beta e^{i \gamma}\right)\left[(1-\lambda) \frac{z f^{\prime}(z)}{f(z)}+\lambda\left(1+\frac{z f^{\prime \prime}(z)}{f^{\prime}(z)}\right)\right]-\beta e^{i \gamma} \prec \frac{1+A z}{1+B z}(z \in U)
$$

and

$$
\left(1+\beta e^{i \gamma}\right)\left[(1-\lambda) \frac{w g^{\prime}(w)}{g(w)}+\lambda\left(1+\frac{w g^{\prime \prime}(w)}{g^{\prime}(w)}\right)\right]-\beta e^{i \gamma} \prec \frac{1+A \omega}{1+B \omega}(\omega \in U),
$$

where $g=f^{-1}$.

Using the parameter setting of Definition 14 in Theorems 3 and 4, respectively, we get the following corollaries.

Corollary 19. Let the function $f \in S_{\sigma}(\lambda, \beta, \gamma, A, B)$ be given by (1). If $a_{k}=0$ for $2 \leq k \leq n-1$, then

$$
\left|a_{n}\right| \leq \frac{(A-B)}{(n-1)[1+\lambda(n-1)]\left|1+\beta e^{i \gamma}\right|}, \quad n \geq 3 .
$$

Corollary 20. If the function $f \in \sigma$ given by (1) be in the class $S_{\sigma}(\lambda, \beta, \gamma, A, B)$, then

$$
\left|a_{2}\right| \leq\left\{\begin{array}{c}
\frac{(A-B)}{\sqrt{\left|1+\beta e^{i \gamma}\right|(1+\lambda)(A-B)+\left|1+\beta e^{i \gamma}\right|^{2}(1-B)}}(B \geq 0) \\
\frac{(A-B)}{\sqrt{\left|1+\beta e^{i \gamma}\right|(1+\lambda)(A-B)+\left|1+\beta e^{i \gamma}\right|^{2}(1+B)}}(-1 \leq B<0)
\end{array}\right.
$$

and

$$
\left|a_{3}-a_{2}^{2}\right| \leq \frac{A-B}{2(1+2 \lambda)\left|1+\beta e^{i \gamma}\right|}
$$

Remark 4. If we put $\beta=\lambda=0$ in Corollaries 19 and 20, then we obtain the results of Hamidi and Jahangiri [49]

If we set $\phi(z)=\sqrt{1+z}$ in Definition 4 of the bi-univalent function class $S_{\sigma}(\lambda, \beta, \gamma, \phi)$, we obtain a new class $S_{L \sigma}(\lambda, \beta, \gamma)$ given by Definition 15 below.

Definition 15. A function $f \in \sigma$ given by (1) is said to be in the class $S_{L \sigma}(\lambda, \beta, \gamma)$ if the following conditions are satisfied:

$$
\left|\left(\left(1+\beta e^{i \gamma}\right)\left[(1-\lambda) \frac{z f^{\prime}(z)}{f(z)}+\lambda\left(1+\frac{z f^{\prime \prime}(z)}{f^{\prime}(z)}\right)\right]-\beta e^{i \gamma}\right)^{2}-1\right|<1(z \in U)
$$


and

$$
\left|\left(\left(1+\beta e^{i \gamma}\right)\left[(1-\lambda) \frac{w g^{\prime}(w)}{g(w)}+\lambda\left(1+\frac{w g^{\prime \prime}(w)}{g^{\prime}(w)}\right)\right]-\beta e^{i \gamma}\right)^{2}-1\right|<1(\omega \in U),
$$

where $g=f^{-1}$.

Using the parameter setting of Definition 15 in Theorems 3 and 4, respectively, we get the following corollaries.

Corollary 21. Let the function $f \in S_{L \sigma}(\lambda, \beta, \gamma)$, be given by (1). If $a_{k}=0$ for $2 \leq k \leq n-1$, then

$$
\left|a_{n}\right| \leq \frac{1}{2(n-1)[1+\lambda(n-1)]\left|1+\beta e^{i \gamma}\right|}, \quad n \geq 3 .
$$

Corollary 22. If the function $f \in \sigma$ given by (1) be in the class $S_{L \sigma}(\lambda, \beta, \gamma)$, then

$$
\left|a_{2}\right| \leq \frac{1}{\sqrt{2\left|1+\beta e^{i \gamma}\right|(1+\lambda)+3\left|1+\beta e^{i \gamma}\right|^{2}(1+\lambda)^{2}}}
$$

and

$$
\left|a_{3}-a_{2}^{2}\right| \leq \frac{1}{4(1+2 \lambda)\left|1+\beta e^{i \gamma}\right|}
$$

If we set $\phi(z)=z+\sqrt{1+z^{2}}$ in Definition 4 of the bi-univalent function class $S_{\sigma}(\lambda, \beta, \gamma, \phi)$, we obtain a new class $S_{\sigma}^{\Delta}(\lambda, \beta, \gamma)$ given by Definition 16 below.

Definition 16. A function $f \in \sigma$ given by (1) is said to be in the class $S_{\sigma}^{\Delta}(\lambda, \beta, \gamma)$ if the following conditions are satisfied:

$$
\begin{aligned}
& \left|\left(\left(1+\beta e^{i \gamma}\right)\left[(1-\lambda) \frac{z f^{\prime}(z)}{f(z)}+\lambda\left(1+\frac{z f^{\prime \prime}(z)}{f^{\prime}(z)}\right)\right]-\beta e^{i \gamma}\right)^{2}-1\right| \\
& <2\left|\left(1+\beta e^{i \gamma}\right)\left[(1-\lambda) \frac{z f^{\prime}(z)}{f(z)}+\lambda\left(1+\frac{z f^{\prime \prime}(z)}{f^{\prime}(z)}\right)\right]-\beta e^{i \gamma}\right|(z \in U)
\end{aligned}
$$

and

$$
\begin{aligned}
& \left|\left(\left(1+\beta e^{i \gamma}\right)\left[(1-\lambda) \frac{w g^{\prime}(w)}{g(w)}+\lambda\left(1+\frac{w g^{\prime \prime}(w)}{g^{\prime}(w)}\right)\right]-\beta e^{i \gamma}\right)^{2}-1\right| \\
& <2\left|\left(1+\beta e^{i \gamma}\right)\left[(1-\lambda) \frac{w g^{\prime}(w)}{g(w)}+\lambda\left(1+\frac{w g^{\prime \prime}(w)}{g^{\prime}(w)}\right)\right]-\beta e^{i \gamma}\right|(\omega \in U)
\end{aligned}
$$

where $g=f^{-1}$.

Using the parameter setting of Definition 9 in Theorems 3 and 4, respectively, we get the following corollaries.

Corollary 23. Let the function $f \in S_{\sigma}^{\Delta}(\lambda, \beta, \gamma)$, be given by (1). If $a_{k}=0$ for $2 \leq k \leq n-1$, then

$$
\left|a_{n}\right| \leq \frac{1}{(n-1)[1+\lambda(n-1)]\left|1+\beta e^{i \gamma}\right|}, \quad n \geq 3 .
$$


Corollary 24. If the function $f \in \sigma$ given by (1) be in the class $S_{\sigma}^{\Delta}(\lambda, \beta, \gamma)$, then

$$
\left|a_{2}\right| \leq \sqrt{\frac{2}{2\left|1+\beta e^{i \gamma}\right|(1+\lambda)+\left|1+\beta e^{i \gamma}\right|^{2}(1+\lambda)^{2}}}
$$

and

$$
\left|a_{3}-a_{2}^{2}\right| \leq \frac{1}{2(1+2 \lambda)\left|1+\beta e^{i \gamma}\right|}
$$

If we set $\phi(z)=\frac{1}{(1-z)^{s}}$ in Definition 4 of the bi-univalent function class $S_{\sigma}(\lambda, \beta, \gamma, \phi)$, we obtain a new class $S_{\sigma}(\lambda, \beta, \gamma, s)$ given by Definition 17 below.

Definition 17. Let $0<s \leq 1$. A function $f \in \sigma$ given by (1) is said to be in the class $S_{\sigma}(\lambda, \beta, \gamma, s)$, if the following conditions are satisfied:

$$
\left(1+\beta e^{i \gamma}\right)\left[(1-\lambda) \frac{z f^{\prime}(z)}{f(z)}+\lambda\left(1+\frac{z f^{\prime \prime}(z)}{f^{\prime}(z)}\right)\right]-\beta e^{i \gamma} \prec \frac{1}{(1-z)^{s}}(z \in U)
$$

and

$$
\left(1+\beta e^{i \gamma}\right)\left[(1-\lambda) \frac{w g^{\prime}(w)}{g(w)}+\lambda\left(1+\frac{w g^{\prime \prime}(w)}{g^{\prime}(w)}\right)\right]-\beta e^{i \gamma} \prec \frac{1}{(1-\omega)^{s}}(\omega \in U),
$$

where $g=f^{-1}$.

Using the parameter setting of Definition 17 in Theorems 3 and 4, respectively, we get the following corollaries.

Corollary 25. Let the function $f \in S_{\sigma}(\lambda, \beta, \gamma, s)$ be given by (1). If $a_{k}=0$ for $2 \leq k \leq n-1$, then

$$
\left|a_{n}\right| \leq \frac{s}{(n-1)[1+\lambda(n-1)]\left|1+\beta e^{i \gamma}\right|}, \quad n \geq 3 .
$$

Corollary 26. If the function $f \in \sigma$ given by (1) be in the class $S_{\sigma}(\lambda, \beta, \gamma, s)$, then

$$
\left|a_{2}\right| \leq \frac{\sqrt{2} s}{\sqrt{\left|1+\beta e^{i \gamma}\right|(1+\lambda) 2 s+\left|1+\beta e^{i \gamma}\right|^{2}(1+\lambda)^{2}(1-s)}}
$$

and

$$
\left|a_{3}-a_{2}^{2}\right| \leq \frac{s}{2(1+2 \lambda)\left|1+\beta e^{i \gamma}\right|}
$$

If we set $\phi(z)=e^{z}$ in Definition 4 of the bi-univalent function class $S_{\sigma}(\lambda, \beta, \gamma, \phi)$, we obtain a new class $S_{\sigma e}(\lambda, \beta, \gamma)$ given by Definition 18 below.

Definition 18. A function $f \in \sigma$ given by (1) is said to be in the class $S_{\sigma e}(\lambda, \beta, \gamma)$, if the following conditions are satisfied:

$$
\left|\log \left(\left(1+\beta e^{i \gamma}\right)\left[(1-\lambda) \frac{z f^{\prime}(z)}{f(z)}+\lambda\left(1+\frac{z f^{\prime \prime}(z)}{f^{\prime}(z)}\right)\right]-\beta e^{i \gamma}\right)\right|<1(z \in U)
$$

and

$$
\left|\log \left(\left(1+\beta e^{i \gamma}\right)\left[(1-\lambda) \frac{w g^{\prime}(w)}{g(w)}+\lambda\left(1+\frac{w g^{\prime \prime}(w)}{g^{\prime}(w)}\right)\right]-\beta e^{i \gamma}\right)\right|<1(\omega \in U),
$$


where $g=f^{-1}$.

Using the parameter setting of Definition 18 in Theorems 3 and 4, respectively, we get the following corollaries.

Corollary 27. Let the function $f \in S_{\sigma}(\lambda, \beta, \gamma, s)$, be given by (1). If $a_{k}=0$ for $2 \leq k \leq n-1$, then

$$
\left|a_{n}\right| \leq \frac{1}{(n-1)[1+\lambda(n-1)]\left|1+\beta e^{i \gamma}\right|}, \quad n \geq 3 .
$$

Corollary 28. If the function $f \in \sigma$ given by (1) be in the class $S_{\sigma}(\lambda, \beta, \gamma, s)$, then

$$
\left|a_{2}\right| \leq \sqrt{\frac{2}{2\left|1+\beta e^{i \gamma}\right|(1+\lambda)+\left|1+\beta e^{i \gamma}\right|^{2}(1+\lambda)^{2}}}
$$

and

$$
\left|a_{3}-a_{2}^{2}\right| \leq \frac{1}{2(1+2 \lambda)\left|1+\beta e^{i \gamma}\right|}
$$

Author Contributions: All authors contributed equally to writing of this paper. All authors have read and agreed to the published version of the manuscript.

Funding: This research has been funded by Scientific Research Deanship at University of Hai'l- Saudi Arabia through project number RG-20020.

Institutional Review Board Statement: Not applicable.

Informed Consent Statement: Not applicable.

Data Availability Statement: Not applicable.

Conflicts of Interest: The authors declare no conflict of interest.

\section{References}

1. Faber, G. Über polynomische Entwickelungen. Math. Ann. 1903, 57, 389-408. ISSN 0025-5831. [CrossRef]

2. Curtiss, J.H. Faber Polynomials and the Faber Series. Am. Math. Mon. Math. Assoc. Am. 1971, 78, 577-596. ISSN $0002-9890$. [CrossRef]

3. Faber, G. Über Tschebyscheffsche Polynome. J. Reine Angew. Math. 1919, 150, 79-106. ISSN 0075-4102. (In German)

4. Grunsky, H. Koeffizientenbedingungen für schlicht abbildende meromorphe Funktionen. Math. Z. 1939, 45, 29-61. ISSN 0025-5874. [CrossRef]

5. Schur, I. On Faber polynomials. Am. J. Math. 1945, 67, 33-41. ISSN 0002-9327. [CrossRef]

6. Suetin, P.K. Series of Faber Polynomials; Analytical Methods and Special Functions, 1; Gordon and Breach Science Publishers: New York, NY, USA, 1998; ISBN 978-90-5699-058-9.

7. Suetin, P.K. Faber Polynomials; Encyclopedia of Mathematics; EMS Press: Berlin, Germany, 2001.

8. Duren, P.L. Univalent Functions, Grundlehren Math. Wissenschaften, Band 259; Springer: New York, NY, USA; Berlin/Heidelberg, Germany; Tokyo, Japan, 1983.

9. Airault, H.; Bouali, A. Di erential calculus on the Faber polynomials. Bull. Sci. Math. 2006, 130, 179-222. [CrossRef]

10. Bouali, A. Faber polynomials, Cayley-Hamilton equation and Newton symmetric functions. Bull. Sci. Math. 2006, 130, 49-70. [CrossRef]

11. Airault, H.; Ren, J. An algebra of di erential operators and generating functions on the set of univalent functions. Bull. Sci. Math 2002, 126, 343-367. [CrossRef]

12. Ma, W.C.; Minda, D. A unified treatment of some special classes of univalent functions. In Proceedings of the Conference on ComplexAnalysis, Tianjin, China, 19-23 June 1992; pp. 157-169.

13. Sokol, J.; Stankiewicz, J. Radius of convexity of some subclasses of strongly starlike functions. Zesz. Nauk. Politech. Rzesz. Mat. 1996, 19, 101-105.

14. Raina, R.K.; Sokol, J. Some properties related to a certain class of starlike functions. C. R. Math. Acad. Sci. Paris 2015, 353, 973-978. [CrossRef]

15. Kanas, S.; Masih, V.S.; Ebadian, A. Relations of a planar domain bounded by hyperbola with family of holomorphic functions. J. Inequalities Appl. 2019, 246, 1-14. [CrossRef] 
16. Mendiratta, R.; Nagpal, S.; Ravichandran, V. On a subclass of strongly starlike functions associated with exponential function. Bull. Malays. Math. Sci. Soc. 2015, 38, 365-386. [CrossRef]

17. Goel, P.; Kumar, S.S. Certain class of starlike functions associated with modified sigmoid function. Bull. Malays. Math. Sci. Soc. 2020, 43, 957-991. [CrossRef]

18. Shams, S.; Kulkarni, S.R.; Jahangiri, J.M. Classes of uniformly starlike and convex functions. Int. J. Math. Math. Sci. 2004, 55, 2959-2961. [CrossRef]

19. Hussain, S.; Rasheed, A.; Zaighum, M.A.; Darus, M. A Subclass of Analytic Functions Related to k-Uniformly Convex and Starlike Functions. J. Funct. Spaces 2017, 2017, 9010964. [CrossRef]

20. Aouf, M.K.; Hossen, H.M.; Lasahin, A.Y. On certain families of analytic functions with negative coefficients. Indian J. Pure Appl. Math. 2000, 31, 999-1015.

21. Lewin, M. On a coefficient problem for bi-univalent functions. Proc. Am. Math. Soc. 1967, 18, 63-68. [CrossRef]

22. Altınkaya, S. Bounds for a new subclass of bi-univalent functions subordinate to the Fibonacci numbers. Turk. J. Math. 2020, 44, 553-560.

23. Altınkaya, S.; Yalcın, S. Faber polynomial coefficient bounds for a subclass of bi-univalent functions. Comptes Rendus Math. 2015, 353, 1075-108. [CrossRef]

24. Aouf, M.K.; El-Ashwah, R.M.; Abd-Eltawab, A.M. New subclasses of bi-univalent functions involving Dziok-Srivastava operator. Int. Sch. Res. Not. 2013, 2013, 387178.

25. Bulut, S. Coefficient estimates for new subclasses of analytic and bi-univalent functions defined by Al-Oboudi differential operator. J. Funct. Spaces Appl. 2013, 2013, 181932. [CrossRef]

26. Bulut, S. Coefficient estimates for a new subclass of analytic and bi-univalent functions defined by Hadamard product. J. Complex Anal. 2014, 2014, 302019. [CrossRef]

27. Caglar, M.; Orhan, H.; Yagmur, N. Coefficient bounds for new subclasses of bi-univalent functions. Filomat 2013, $27,1165-1171$. [CrossRef]

28. Deniz, E. Certain subclasses of bi-univalent functions satisfying subordinate conditions. J. Class. Anal. 2013, 2, 49-60. [CrossRef]

29. Hayami, T.; Owa, S. Coefficient bounds for bi-univalent functions. Pan-Am. Math. J. 2012, 22, 15-26.

30. Jahangiri, J.M.; Hamidi, S.G. Faber polynomial coefficient estimates for analytic bi-bazilevic functions. Mat. Vesn. 2015, 67, 123-129.

31. Lashin, A.Y. On certain subclasses of analytic and bi-univalent functions. J. Egypt. Math. Soc. 2016, 24, 220-225. [CrossRef]

32. Lashin, A.Y. Coefficient estimates for two subclasses of analytic and bi-univalent functions. Ukr. Math. J. 2019, 70, 1484-1492. [CrossRef]

33. Lashin, A.Y.; L-Emam, F.Z.E. Faber polynomial coefficients for certain subclasses of analytic and biunivalent functions. Turk. J. Math. 2020, 44, 1345-1361. [CrossRef]

34. Magesh, N.; Rosy, T.; Varma, S. Coefficient estimate problem for a new subclass of bi-univalent functions. J. Complex Anal. 2013, 2013, 474231.

35. Magesh, N.; Yamini, J. Coefficient bounds for certain subclasses of bi-univalent functions. Int. Math. Forum. 2013, 8, 1337-1344. [CrossRef]

36. Murugusundaramoorthy, G.; Magesh, N.; Prameela, V. Coefficient bounds for certain subclasses of bi-univalent function. Abstr Appl. Anal. 2013, 2013, 573017. [CrossRef]

37. Peng, Z.-G.; Han, Q.-Q. On the coefficients of several classes of bi-univalent functions. Acta Math. Sci. Ser. B Engl. Ed. 2014, 34, 228-240. [CrossRef]

38. Porwal, S.; Darus, M. On a new subclass of bi-univalent functions. J. Egypt. Math. Soc. 2013, 21, 190-193. [CrossRef]

39. Srivastava, H.M.; Bulut, S.; Caglar, M.; Yagmur, N. Coefficient estimates for a general subclass of analytic and bi-univalent functions. Filomat 2013, 27, 831-842. [CrossRef]

40. Srivastava, H.M.; Murugusundaramoorthy, G.; Magesh, N. Certain subclasses of bi-univalent functions associated with the Hohlov operator. Glob. J. Math. Anal. 2013, 1, 67-73.

41. Srivastava, H.M.; Murugusundaramoorthy, G.; Vijaya, K. Coefficient estimates for some families of bi-Bazilevic functions of the Ma-Minda type involving the Hohlov operator. J. Class. Anal. 2013, 2, 167-181. [CrossRef]

42. Srivastava, H.M.; Motamednezhad, A.; Adegani, E.A. Faber Polynomial Coefficient Estimates for bi-univalent functions defined by using differential subordina-tion and a certain fractional derivative operator. Mathematics 2020, 8, 172. [CrossRef]

43. Tang, H.; Deng, G.-T.; Li, S.-H. Coefficient estimates for new subclasses of Ma-Minda bi-univalent functions. J. Inequalities Appl. 2013, 2013, 317. [CrossRef]

44. Agarwal, P.; Nieto, J.J. Some fractional integral formulas for the Mittag-Leffler type function with four parameters. Open Math 2015, 1, 537-546. [CrossRef]

45. Agarwal, P.; Chand, M.; Baleanu, D.; O'Regan, D.; Jain, S. On the solutions of certain fractional kinetic equations involving k-Mittag-Leffler function. Adv. Differ. Equ. 2018, 249, 13. [CrossRef]

46. Alderremy, A.A.; Saad, K.M.; Agarwal, P.; Aly, S.; Jain, S. Certain new models of the multi space-fractional Gardner equation. Phys. A 2020, 545, 11. [CrossRef]

47. Saoudi, K.; Agarwal, P.; Kumam, P.; Ghanmi, A.; Thounthong, P. The Nehari manifold for a boundary value problem involving Riemann-Liouville fractional derivative. Adv. Differ. Equ. 2018, 263, 18. [CrossRef] 
48. Darwish, H.E.; Lashin, A.Y.; El-Ashwah, R.M.; Madar, E.M. Coefficient estimates of some classes of rational functions. Int. J. Open Probl. Complex Anal. 2019, 11, 16-30. [CrossRef]

49. Hamidi, S.G.; Jahangiri, J.M. Faber polynomial coefficients of bi-subordinate functions. Comptes Rendus Math. 2016, 354, 365-370. [CrossRef]

50. Goyal, S.P.; Kumar, R. Coefficient estimates and quasi-subordination properties associated with certain subclasses of analytic and bi-univalent functions. Math. Slovaca 2015, 65, 533-544. [CrossRef]

51. Zireh, A.; Adegani, E.A.; Bidkham, M. Faber polynomial coefficient estimates for subclass of bi-univalent functions defined by quasi-subordinate. Math. Slovaca 2018, 68, 369-378. [CrossRef]

52. Deniz, E.; Jahangiri, J.M.; Hamidi, S.G.; Kina, S.K. Faber polynomial coefficients for generalized bi-subordinate functions of complex order. J. Math. Inequalities 2018, 12, 645-653. [CrossRef] 